\title{
Thermodynamic derivation of a non-linear poroelastic model describing hemodynamics- mechanics interplay in the lamina cribrosa
}

Filippo Recrosi ${ }^{1}$, Rodolfo Repetto ${ }^{2}$, Amabile Tatone ${ }^{3}$, Giovanna Guidoboni ${ }^{4}$

${ }^{1}$ GSSI, Gran Sasso Science Institute, L'Aquila, Italy, ${ }^{2}$ IICCA, University of Genoa, Genoa, Italy, ${ }^{3} \mathrm{DISIM}$, University of L'Aquila, L'Aquila, Italy, ${ }^{4}$ Department of Electrical Engineering and Computer Science, University of Missouri, Columbia, MO, USA.

\section{Abstract}

In this paper we formulate a poroelastic model starting from a model of species diffusion in an elastic material. The model is applied to study the mechanics of the lamina cribrosa (LC) in the eye. The LC is a porous tissue at the head of the optic nerve. Deformation of this tissue and impairment of blood flow induced by tissue deformation are considered to be related to the pathogenesis of glaucoma.

The governing equations are derived from general thermomechanical principles. We carefully revise the role of the energy-stress Eshelby tensor, mutuated from the framework of tissue growth, in describing the hemo-mechanical behaviour of the tissue.

The model accounts for non-linear deformations of the solid matrix and deformation-induced changes in porosity and permeability. The model provides a qualitative better undertanding of the phatophysiology and pathogenesis of glaucoma in terms of coupling between tissue deformation and the resulting impaired hemodynamics inside the LC.

Keywords: blood perfusion, large deformations, poroelasticity, species diffusion

Correspondence: Filippo Recrosi, via Francesco Crispi 7, L’Aquila, Italy.

E-mail: filippo.recrosi@gssi.infn.it 


\section{Introduction}

The LC is a part of the optic nerve head $(\mathrm{ONH})$ acting as a scaold for collecting the retinal ganglion cells (RGC) axons. The optic nerve carries, through the axons, the signal generated by the retina to the brain. The LC is also a region crucial for blood supply to the whole optic nerve. An increased pressure inside the vitreous chamber (intraocular pressure, IOP) or a decreased pressure inside the subarachnoid space surrounding the optic nerve (cerebrospinal fluid pressure, CSFp) can generate a mechanical deformation of the LC, pinching the RGCs, possibly progressively leading to cell death. Further, lamina deformation can have an adverse effect on vascular perfusion of ocular tissue. Damages of the optic nerve are typical of glaucoma, a progressive optic neuropathy resulting in irreversible vision loss and blindness. A mathematical model able to describe the mechanics behind the pathogenesis of glaucoma would be invaluable, not only for diagnostic purposes by providing useful simulations and data, but also to support and guide, in the near future, the design of medical devices. Many researchers have worked on the mechanics of the $\mathrm{ONH}$ accounting for the complicated structure of the lamina. However, most of the literature focuses on the lamina deformation without accounting for the interactions with blood perfusion. ${ }^{1-3} \mathrm{~A}$ possible strategy to eectively couple tissue deformation and blood perfusion is to model the tissue as a poroelastic material, where the saturated porosity stands for the vascular network made up of small vessels. ${ }^{4,5}$ The aim of the present work is to study the interaction between porosity, tissue deformation, and blood perfusion.

\section{Methods}

We derive a poroelastic model ${ }^{6,7}$ from a model of species diusion in an elastic material..$^{8,9}$ This derivation is formulated in the not common framework of virtual powers, ${ }^{10}$ and it is carried out by transforming, in a natural way, quantities appropriate to a diusing species, such as concentration, chemical potential, and molar flux into the corresponding quantities which are appropriate to a saturated porous material, such as porosity, interstitial pressure, and discharge, while retaining the usual description for a non-linear elastic solid, based on deformation and stress.

We get a couple of power balance laws for the forces and for the mass of the fluid filling the pores:

$$
\begin{aligned}
& \int_{P_{0}} \mathbf{b}_{0} \cdot v_{0} d V+\int_{\partial P_{0}} \mathbf{t}_{0} \cdot \mathbf{v}_{0} d A=\int_{P_{0}} \mathbf{S}_{0} \cdot \dot{\mathbf{F}}_{0} d V \\
& \int_{P_{0}} \breve{p}_{0} \dot{\beta} d V=-\int_{\partial P_{0}} \breve{p}_{0} \mathbf{q}_{0} \cdot \mathbf{n}_{0} d A+\int_{P_{0}} \mathbf{q}_{0} \cdot \nabla \breve{p}_{0} d V
\end{aligned}
$$


where $P_{0}$ is any part of the referential body simultaneously showing the presence of both fluid and solid parts, $\mathrm{v}_{0}$ is the velocity test field, $\dot{\mathbf{F}}_{0}$ is the time rate of the deformation gradient, $\mathbf{b}_{0}$ and to are respectively any volume and surface density of external forces, and $\mathbf{S}_{0}$ is the first Piola-Kirchho stress tensor. Moreover, $\breve{p}_{0}$ is the interstitial pore pressure, $\beta$ is the determinant of the total deformation and $\mathbf{q}_{0}$ is the fluid flux.

Superimposing the contributions of both external power expenditures, the energy imbalance principle reads:

$$
\mathbf{S}_{0} \cdot \dot{\mathbf{F}}_{0}+\breve{p}_{0} \dot{\beta}-\mathbf{q}_{0} \cdot \nabla \breve{p}_{0}-\frac{d}{d t} \varphi \geq 0
$$

from which we state some constitutive prescriptions on the form of every dissipative term, together with a Darcy's law coupling tissue deformation with the flux:

$$
\mathrm{q}_{0}=-\mathbf{K}_{0} \nabla \widetilde{p}_{0}
$$

We assume a second order dependence of the permeability tensor $\mathbf{K}_{0}$ from the porosity, through a modified Carman-Kozeny equation, which should be appropriate to describe the capillary network. We emphasize how this characterization relies on the expression for the free energy, mutuated by tissue growth models, and in turn, on the stress energy function:

$$
\mathbf{T}=\widehat{\mathbf{T}}^{\text {eff }}\left(\mathbf{F}_{1}, \beta\right)-\breve{p}_{0} \mathbf{I} \mid=\left(\widehat{\mathbf{T}}^{\mathrm{g}}\left(\mathbf{F}_{1}\right)+\left(\widehat{\mathrm{p}}_{\phi}(\beta)+\phi_{1}\left(\mathbf{F}_{1}\right) \mathbf{I}\right)-\widehat{p}_{0} \mathbf{I}\right)
$$

Table 1. Parameter values used in the simulations.

\begin{tabular}{|l|l|}
\hline Description & Value \\
\hline lamina thickness & $0.02 \mathrm{~cm}$ \\
\hline lamina external radius & $0.095 \mathrm{~cm}$ \\
\hline central retinal vessel passage radius & $0.01 \mathrm{~cm}$ \\
\hline shear modulus of the lamina & $8 \times 10^{3} \mathrm{~Pa}$ \\
\hline bulk modulus of the lamina & $6 \times 10^{5} \mathrm{~Pa}$ \\
\hline shear modulus of the sclera & $11 \times 10^{5} \mathrm{~Pa}$ \\
\hline reference porosity & 0.01 \\
\hline max IOP & $4666-4800 \mathrm{~Pa}$ \\
\hline RLTp & $1300 \mathrm{~Pa}$ \\
\hline ciliary pressure & $2000 \mathrm{~Pa}$ \\
\hline central vein pressure & $1000 \mathrm{~Pa}$ \\
\hline
\end{tabular}


where $\widehat{\mathbf{T}}^{\text {eff }}$ is the ective stress and $\phi_{1}\left(\mathbf{F}_{1}\right)$ is the spherical part of the Eshelby tensor. ${ }^{11}$

We perform numerical simulations on a three-D system - depicted in the background of Figure 1 - consisting in a curved disk embedded in a portion of peripapillary slera (ppS). The LC is modelled as a non-linear poroelastic tissue, whose governing equations are (4) and (5), while the surrounding ppS is described as an hyperelastic incompressible medium. The blood supply is imposed by assigning the value of the ciliary pressure at the LC-ppS interface, aswell as the blood drainage by assigning the value of the central vein pressure on the hole surface, located at the center of the cap (Fig. 1). On the upper boundary of the LC (facing the vitreous chamber), we increase the IOP from physiological values ( $15 \mathrm{mmHg}$ ) to pathological ones (up to $35 \mathrm{mmHg}$ ), whereas on the lower surface (facing to the retrolaminar region) we assign a value of retrolaminar tissue pressure (RLTp). The most meaningful parameter values are reported in Table 1.

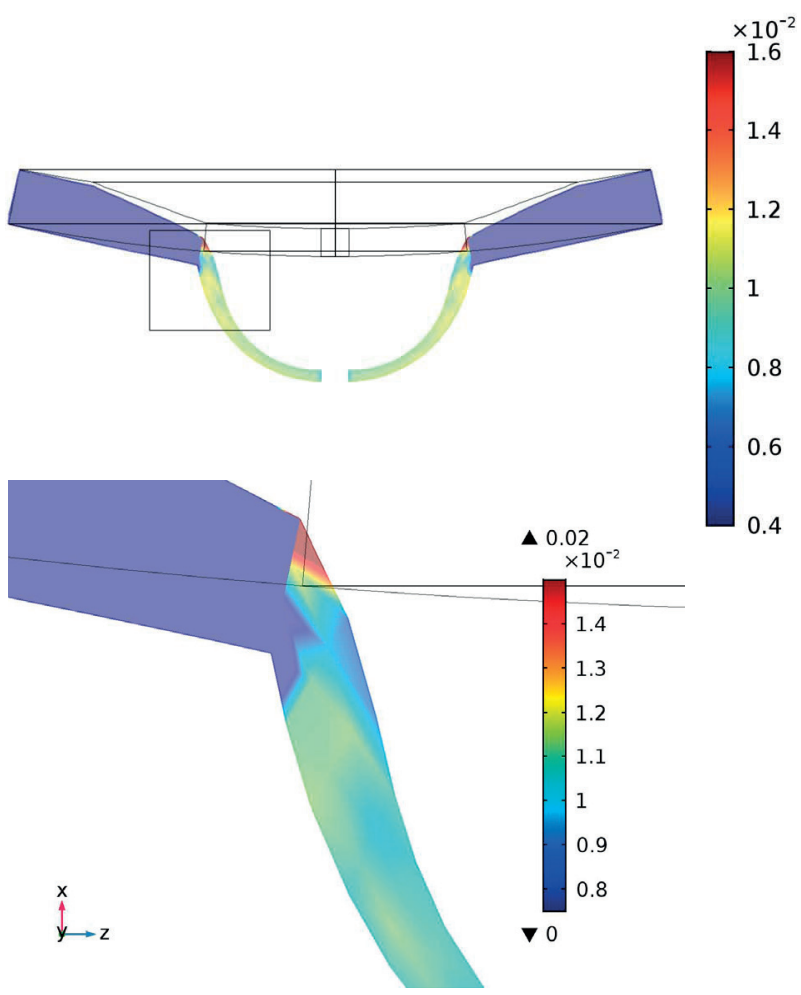

Fig. 1. Radial section of the LC-ppS system deformed under IOP $=35 \mathrm{mmHg}$. The colors show the value of the porosity field inside the LC: from low (blue) to high values (red). The boxed picture is a magnification of the interface region. 


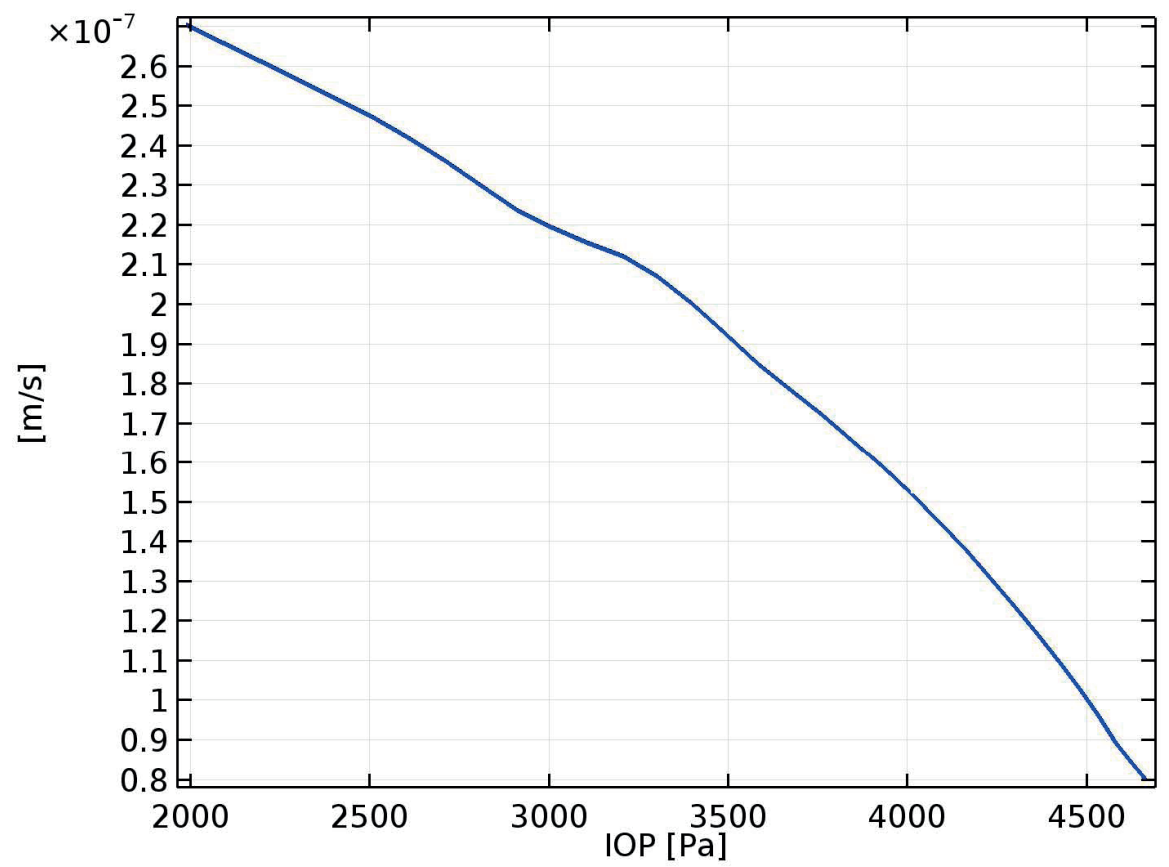

Fig. 2. Blood flux as a function of IOP.

\section{Results}

Figure 1 shows the porosity map, obtained for a high IOP value $(35 \mathrm{mmHg})$, and the corresponding tissue deformation. A magnification of the LC-pps interface region is also shown, since this is where the most significant coupling between tissue deformation and changes in porosity occur, which strongly aects blood flow.

Figure 2 shows that the blood flux decreases for increasing values of IOP. IOP increases from physiological values (corresponding to $2000 \mathrm{~Pa}$ ) towards increasingly more severe stages of glaucoma, up to IOP $=4666 \mathrm{~Pa}$. The cause of such a decrease is related to the large deflection of LC portions close to the sclera, and the related appearance of a low porosity region crossing all the LC thickness (see the magnification of the interface region in Fig. 1).

\section{Discussion}

These preliminary results suggest that tissue deformation can influence blood flow significantly, at least in certain regions of the LC. The decrease in blood flow from 
posterior ciliary arteries to the central retinal vein secondary to tissue deformation is far from being a minor adverse eect on the RGC axons, and it seems to be crucial for better understanding the pathogenesis of glaucoma.

\section{References}

1. Sigal IA, Flanagan JG, Tertinegg I, Ethier CR. finite element modeling in optic nerve. Invest Ophthalmol Vis Sci. 2004;45(12):4378-4387.

2. Sigal IA, Hongli Y, Roberts MD, Burgoyne CF, Downs CJ. IOP-induced lamina cribrosa displacement and scleral canal expansion: an analysis of factor interactions using parameterized eye-specific models. Invest Ophthalmol Vis Sci. 2011;52(3):1896-1907.

3. Sigal IA, Grimm JL, Jan NJ, Reid K, Minckler DS, Brown DJ. Eye-specific IOP-induced displacements and deformations of human lamina cribrosa. Invest Ophthalmol Vis Sci. 2011;55(1):1-15.

4. Causin P, Guidoboni G, Harris A, Prada D, Sacco R, Terragni S. A poroelastic model for the perfusion of the lamina cribrosa in the optic nerve head. Math Biosci. 2014;(257):33-41.

5. Guidoboni G, Harris A, Carichino L, Arieli Y, Siesky BA. Effect of intraocular pressure on the hemodynamics of the central retinal artery: a mathematical model. Math Biosci Eng. 2014;(11):523-546.

6. Biot MA. General theory of of three-dimensional consolidation. J Appl Phys. 1941;(12):155-164.

7. Biot MA. Theory of finite deformations of porous solids. Ind Univ Math J. 1972;21:597-620.

8. Larchè $F$, Cahn JW. Linear theory of thermomechanical equilibrium solids under stress. Acta. Metall. 1973;21:1051-1063.

9. Larchè F, Cahn JW. The interactions of composition and stress in crystalline solids. J Res Natl Bur Stand. 1984;89:467-500.

10. Honga W, Zhaoa X, Zhoua J, Suo Z. A theory of coupled diusion and large deformation in polymeric gels. J Mechan Phys Solids. 2008;56:1779-1793.

11. Eshelby JD. Elastic energy momentum tensor. J Elasticity. 1975;5:331-335. 